Document downloaded from:

http://hdl.handle.net/10251/121384

This paper must be cited as:

Calabuig Soler, D.; Martín-Sacristán, D.; Monserrat Del Río, JF.; Botsov, M.; Gozálvez Serrano, D. (2018). Distribution of Road Hazard Warning Messages to Distant Vehicles in Intelligent Transport Systems. IEEE Transactions on Intelligent Transportation Systems. 19(4):1152-1165. https://doi.org/10.1109/TITS.2017.2718103

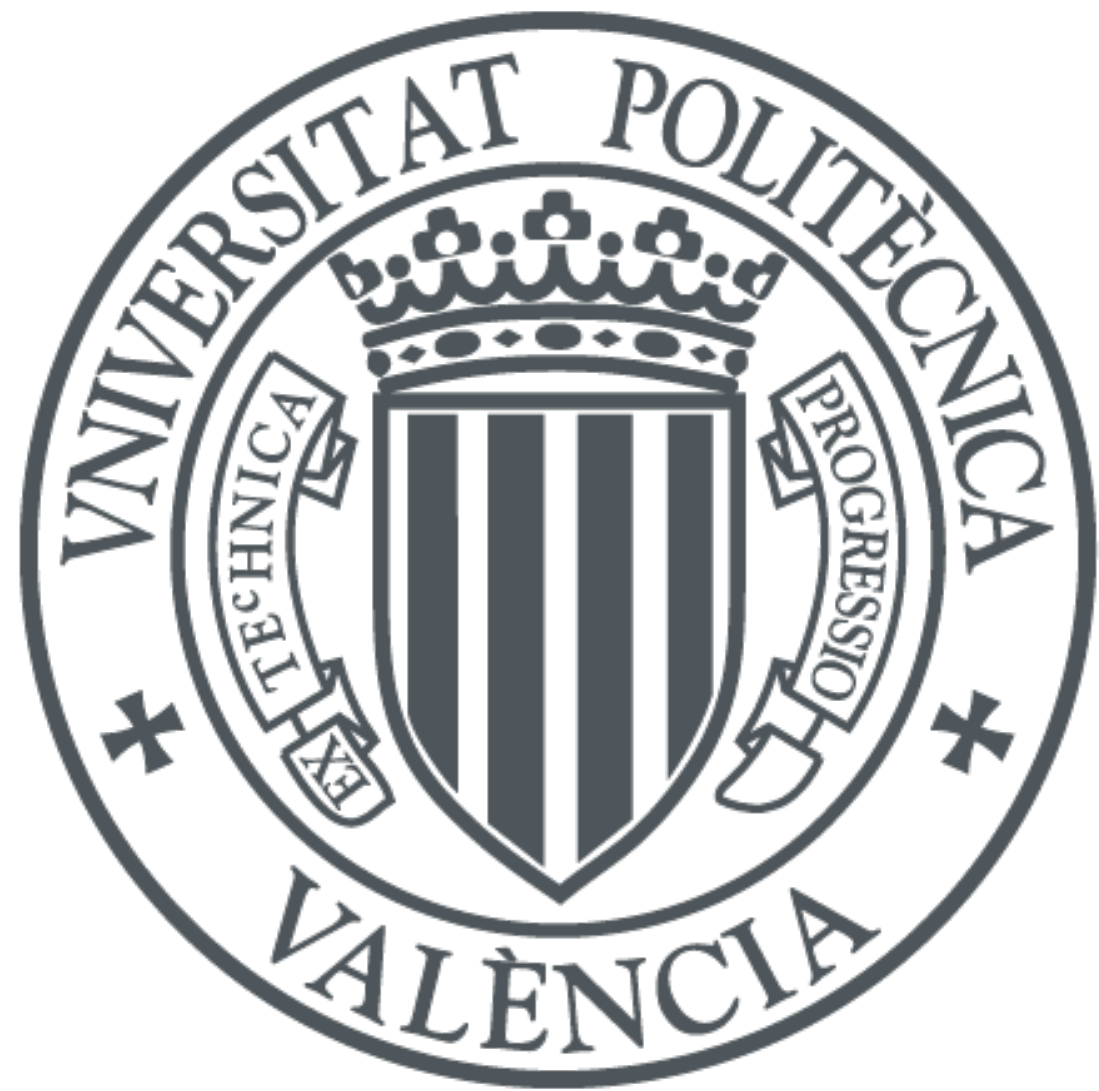

The final publication is available at

http://doi.org/10.1109/TITS.2017.2718103

Copyright Institute of Electrical and Electronics Engineers

Additional Information 


\title{
Distribution of Road Hazard Warning Messages to Distant Vehicles in Intelligent Transport Systems
}

\author{
Daniel Calabuig, Member, IEEE, David Martín-Sacristán, Jose F. Monserrat, Senior Member, IEEE, \\ Mladen Botsov, Student Member, IEEE, and David Gozálvez
}

\begin{abstract}
The efficient distribution of Intelligent Transport System (ITS) messages is fundamental for the deployment and acceptance of ITS applications by mobile network operators and the automotive industry. In particular, the distribution of Road Hazard Warning (RHW) messages to distant vehicles requires special mechanisms. In this case, the combination of direct communication between vehicles and the wide area coverage provided by cellular networks might be crucial not only for reducing the data transmission costs but also for improving the timeliness of ITS information. Moreover, the application of clustering and cluster head selection mechanisms among vehicles can increase the efficiency of hybrid vehicular and cellular communication networks. This paper introduces a novel cluster head selection technique for the distribution of RHW messages, and proposes an implementation of another legacy technique that was originally intended for Mobile Ad-hoc Networks (MANETs). The paper evaluates the performance of these techniques by means of computer simulations in two scenarios with distinct congestion and propagation conditions. The simulation results show the potential benefit of hybrid networks compared to pure cellular transmissions, especially if the novel cluster head selection technique is used.
\end{abstract}

Index Terms-Intelligent transport systems, road hazard warnings, cluster head selection.

\section{INTRODUCTION}

I $\mathrm{N}$ the upcoming years, the transportation industry will witness a fascinating period of major changes and evolution thanks to the implementation of Intelligent Transport Systems (ITS). Probably, one of the most interesting characteristics of ITS is the exchange of information between vehicles in order to increase the traffic awareness of drivers, and even of vehicles in case of highly autonomous driving. In this context, the so-called Road Hazard Warning (RHW) messages can be transmitted upon detection of a hazardous event. Although these messages were primarily thought to be locally distributed, in many cases, distant vehicles can benefit from receiving remotely generated RHW messages, e.g., several kilometers away, so that the driver and the vehicle itself can act accordingly and even modify the route to avoid the hazardous situation. Examples of such cases are notifications of traffic jams on a highway or vehicles driving in the wrong direction.

Daniel Calabuig (dacaso@iteam.upv.es), David Martín-Sacristán (damargan@iteam.upv.es), and Jose F. Monserrat (jomondel@iteam.upv.es) are with the Instituto de Telecomunicaciones y Aplicaciones Multimedia (iTEAM) at the Universitat Politècnica de València (UPV), Camino de Vera s/n, 46022 Valencia, Spain. David Gozálvez (dagoser@alumni.upv.es) is an alumni of the UPV. Mladen Botsov (mladen.botsov@ campus.tu-berlin.de) is a Ph.D. student at the Technische Universität Berlin, Germany.
The European Telecommunications Standards Institute (ETSI) has released a set of standards for addressing Cooperative ITS (C-ITS) [1]. One of these standards is referred to as ITS-G5 [2], which corresponds to the lowest protocol stack layer identified in [1]. This technology, which is based on the IEEE 802.11-2012 specification, was designed for Vehicle to Vehicle (V2V) communications in a Vehicular Ad-hoc Network (VANET).

In VANETs, messages can be broadcasted to distant receivers using multiple transmission hops, which may produce broadcast storms, i.e., extreme amounts of broadcast traffic. Moreover, multi-hop transmissions may not be able to forward the message to all vehicles in areas with a low density of ITS stations, i.e., a vehicle or infrastructure element capable of receiving and transmitting ITS messages. Although some mechanisms have been proposed to alleviate these problems [3], they do not completely solve them, as concluded from the results in [3], and require significant message reception delays.

Indeed, another means to reach distant vehicles and spread the RHW messages to as many ITS stations as possible is the use of certain infrastructure. For instance, interconnected Road Side Units (RSUs) could be used as access points of a supporting infrastructure, as proposed by the so-called CAN DELIVER approach [4]. However, this approach might suffer from coverage problems since RSUs are not deployed for coverage maximization but for their primary functionality related with traffic management. Another example of supporting infrastructure is a cellular network, which might be seen as a better option due to its coverage-centric deployment. In this case, an ITS server, located for instance in the internet, redistributes the RHW messages, i.e., it collects and routes the messages from and to other ITS stations. Moreover, the RHW messages can be distributed using the VANET and the cellular infrastructure interoperating in three different configurations: Cellular Unicast Configuration (CUC), Cellular Broadcast Configuration (CBC), and Hybrid Cellular-VANET Configuration (HCVC). Figure 1 depicts an example of RHW message distribution among vehicles in two perpendicular streets with the three configurations. The distribution procedure comprises four or five steps, depending on the configuration of the VANET and cellular infrastructure interoperation. These steps are the following: an accident happens, the neighboring ITS stations detect a road hazard, the road hazard is signaled to the network, the network transmits a RHW message, and a subset of ITS stations (named cluster heads in Figure 1) distribute the RHW message. The main characteristics of the three configurations are summarized in Table I. 


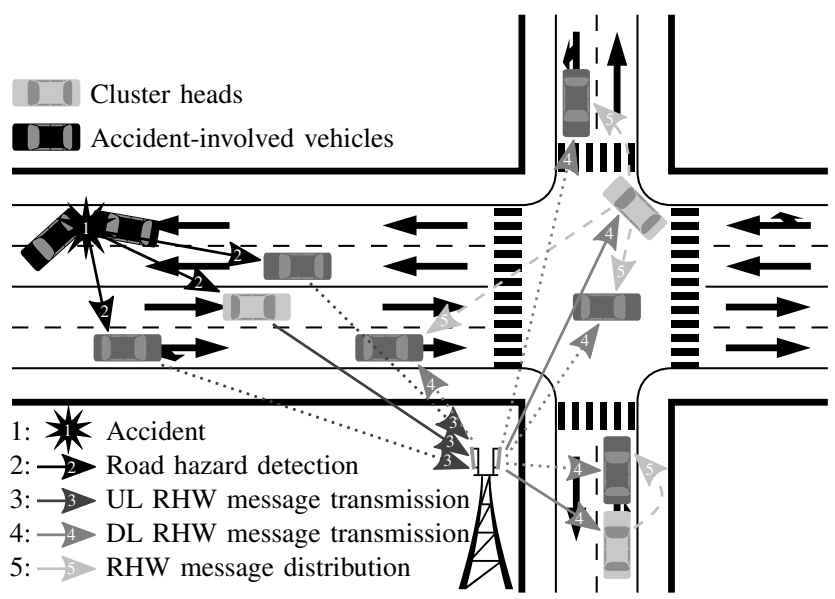

Fig. 1. Distribution of an accident-related RHW message in the three VANET and cellular infrastructure interoperation configurations. Solid arrows represent transmissions that occur in the three configurations. Striped arrows refer to only HCVC transmissions, and dotted arrows to only CUC and/or CBC transmissions (UL in only the CUC, DL in the CUC and $\mathrm{CBC}$ ).

TABLE I

CHARACTERISTICS OF THE VANET AND CELLULAR INFRASTRUCTURE INTEROPERATION CONFIGURATIONS.

\begin{tabular}{|c||c|c|c|}
\hline & CUC & CBC & HCVC \\
\hline Allows UL transmissions & yes & no & yes \\
\hline $\begin{array}{c}\text { Need of cellular broadcasting } \\
\text { features }\end{array}$ & no & yes & no \\
\hline UL load & high & - & low \\
\hline DL load & high & very low & low \\
\hline Size of a user profile database & large & - & small \\
\hline
\end{tabular}

A CUC can be used for both Uplink (UL) and Downlink (DL) transmissions. In this case, all the ITS stations that detect the road hazard send a RHW message to the infrastructure (dotted and solid arrows in dark gray in Figure 1), which filters the different messages about the same event and retransmits only one of them to potentially interested ITS stations (dotted and solid arrows in medium gray in Figure 1). All these transmissions are point-to-point (ptp). The main drawback of this configuration is the large amount of cellular resources that is required to deliver the RHW messages, which, besides, might produce undesirable delays in congested scenarios or with high path losses. In addition to this, the ITS server has to be aware of the existence of all ITS stations to be able to route the messages to them. Therefore, the server has to manage a user profile data base with all the necessary information. In the case of the $\mathrm{CBC}$, all the ITS stations belonging to a broadcast area, defined as any set of cells specified by the cellular operator, are addressed collectively, rather than individually. This implies that keeping a complete user profile in the server is not necessary. Thus, scalability and privacy are less critical and less signaling is foreseen. Although the CBC is more efficient, it exhibits two main drawbacks: (i) cellular operators do not usually activate the broadcasting features of their networks, and (ii) it cannot be used in the UL.

In the case of the HCVC, a subset of ITS stations act as gateways between the infrastructure and the rest of the ITS stations (vehicles in light gray in Figure 1). In particular, in the UL, they capture RHW messages generated by neighboring ITS stations and transmit them to the infrastructure with ptp transmissions (solid arrow in dark gray in Figure 1), which reduces the connection attempts and the amount of traffic transmitted to the network. In the DL, the gateways receive the RHW messages from the infrastructure with ptp transmissions (solid arrows in medium gray in Figure 1) and retransmit them to their neighboring ITS stations by means of direct communication and broadcast transmissions (stripped arrows in light gray in Figure 1). These gateways can be seen as cluster heads of certain ITS station clusters. The use of direct vehicle-to-vehicle communication together with broadcast dissemination improves the efficiency and timeliness of ITS information compared to traditional cellular ptp communication in scenarios with a congested cellular network or with high path losses. Another difference of the HCVC as compared with the CUC and CBC is the potential need for additional signaling between the ITS stations and the cellular network or the ITS server. This signaling is used to e.g. indicate which ITS stations are acting as cluster heads at any moment. The higher the number of cluster head reconfigurations, the higher the signaling.

Clustering and cluster head selection in Mobile Ad-hoc Networks (MANETs) have been widely studied in the literature (see a survey in [5]), although most approaches consider only ptp communications without infrastructure, and the clustering procedures focus on improving some performance indicators of the message routing between a transmitter and a receiver through the MANET. The goal of the envisioned HCVC for RHW messages distribution is completely different. In particular, messages do not have to be delivered to a single receiver, but need to be distributed to all ITS stations in a certain area.

Two main results related to the distribution of RHW messages to distant vehicles are presented in this paper. First, a new class of cluster head selection techniques in which ITS stations become cluster heads with a certain precomputed probability is proposed. The main advantages of this class with respect to those of the literature are summarized in Section II. Second, the performance of the three configurations is analyzed by means of simulations in two scenarios with high and low path losses, respectively. The results show the gain that can be achieved by an HCVC with respect to traditional CUC and CBC scenarios in a cellular network with high path losses.

The rest of the paper is organized as follows. Section II describes the features of good cluster head selection techniques for the purpose of this paper, and analyzes the techniques proposed in the literature with respect to these features. Sections III and IV describe two cluster head selection techniques, one selected from the literature and a new probabilistic technique, respectively. Section V introduces the simulation models and assumptions made in the assessment, and Section VI presents the results and performance analysis. Finally, Section VII highlights notable implications derived from the analysis, and Section VIII draws the main conclusions of the 
paper.

\section{Clunster head selection techniques features} AND LITERATURE REVIEW

For the purpose of this paper, good clustering and/or cluster head selection techniques should

a) be distributed and not require a central managing entity;

b) minimize the traffic load in the VANET by reducing the number and size of the signaling messages transmitted through the VANET for the cluster head selection;

c) have a fast response and adaptation capabilities, in order to cope with the highly variable nature of VANETs;

d) select cluster heads as stable as possible, so that unnecessary cluster head changes do not increase the signaling in the cellular network;

e) maximize the area covered by the cluster heads; and

f) be robust in highly variable environments, where shadowing might cause coverage holes.

As highlighted in the Introduction, cluster head selection techniques proposed in the literature are not designed for broadcasting RHW messages to distant vehicles. As a consequence, they are not able to comply with some of the previous properties. In the rest of this section, we will analyze distributed techniques, which satisfy property a) above, i.e., those without a central managing entity. The distributed techniques proposed in the literature can be classified as follows [6]:

- Lowest-ID (LID) algorithms [7]. LID is a well-known and studied approach in which ITS stations periodically broadcast their ID numbers. If an ITS station has the lowest ID number among its neighbors, it becomes the cluster head. When the ID number is not the lowest one, it is necessary to check if the neighbors with lower IDs are cluster heads or not. If none is a cluster head, the ITS station becomes a cluster head itself.

- Highest-degree algorithms [7]. The goal of these algorithms is to minimize the number of clusters. Each ITS station is aware of the number of neighbors. This number is broadcasted like the ID in the LID algorithm. Then, the ITS station with the highest number of neighbors is selected as the cluster head (in case of a tie, LID prevails).

- Weight-based algorithms. In this family of algorithms, weights are computed for each ITS station and transmitted, so that the station with the highest weight becomes the cluster head (in case of a tie, LID again prevails). In fact, the two previous algorithms may be seen as special cases of weight-based algorithms, wherein the weights are all ones for the LID algorithm, or the number of neighbors for the highest-degree algorithm. In [8], the chosen weights are inversely proportional to the vehicle speed in a quasi-static network (vehicles have a reduced mobility). This approach aims at minimizing the number of cluster head reassignments. In [9], the same approach is extended to any mobile network, without the constraint of quasi-static vehicles. After these two works, the weights computation was extended to include other metrics. In particular, in [10] the weights are computed as a weighted sum of factors that depend on the number of neighbors, the distance to neighbors, speed, and the time during which the vehicle has been acting as a cluster head. Other works have considered the cluster stability in the cluster head selection [6], [11], [12]. In particular, in [6] the concept of stability factor is defined and used as weights for the cluster head selection. A similar approach was used in [11], whereas in [12], vehicles that are expected to remain in a cluster for a longer time are selected as cluster heads. In [13] a dynamic clustering mechanism is proposed. The clustering procedure is divided into three phases, which are based on direction of movement, signal strength of the Universal Mobile Telecommunications System (UMTS) cells, and transmission range, respectively. Then, the first cluster heads are selected in each cluster by measuring the Time-To-Live (TTL) of a packet inside the cluster. The vehicle with the lowest TTL is at the cluster center and, hence, selected as cluster head. The selection of subsequent cluster heads is managed by the current cluster head and done after computing some weights based on mixed criteria. In [14] the work of [13] is extended to consider Long Term Evolution (LTE) and to Quality of Service (QoS) provisioning.

Previous techniques require signaling data interchange among ITS stations to select the cluster heads, which increases the VANET load, and hence do not comply with property b) (minimize signaling) presented above. In particular, ITS stations have to broadcast their IDs (in all previous techniques), the number of neighbors (in the highest degree algorithms) and the weights (in the weight-based algorithms), in addition to other data required to compute the weights, like location [6], [10], [11], [12], [13], [14], stability factor [6], [11], direction of movement [11], [12], [13], [14], received signal strength from the infrastructure [13], [14], and speed [12], [13], [14]. Moreover, in some cases, the signaling is divided into several phases, e.g., [13] and [14], which complicates and delays the selection, and, hence, goes against property c) (fast reselection). If other ITS messages that include the station ID are transmitted through the VANET, the LID algorithm does not increase the VANET load significantly, since the IDs of each station are already known from previous messages. However, the LID algorithm does not take into account cluster head coverage (property e)) or robustness (property f)).

In this paper, a new class of cluster head selection techniques in which ITS stations become cluster heads with a certain precomputed probability is proposed. The main advantage of these techniques is that they are able to combine different parameters that characterize the suitability of a cluster head (similarly to the weights of weight-based algorithms), and, at the same time, they need only one signaling bit (in contrast to weight-based algorithms, which require the interchange of weights among other parameters). This new class, which is described in Section IV, will be evaluated and compared with the LID-based algorithm described in Section III.

\section{LID-BASED CLUSTER HEAD SELECTION TECHNIQUE}

In this section, we describe a LID-based cluster head selection technique that will be used as a reference in the 
results shown in Section VI. With this technique, ITS stations manage a database of neighbor ITS stations with three fields: the neighbor ID, a one-bit cluster head flag, and a timer. We assume that ITS stations periodically send ITS messages that contain their IDs and one bit indicating if they were cluster heads when the message was generated, i.e., the cluster head flag. When one of these messages is received, the receiver ITS station looks for the sender ID in the database, and, if it is found, updates the cluster head flag and sets the timer to zero. If the sender ID is not in the database, it creates a new entry with the sender ID, the cluster head flag indicated in the ITS message, and a new timer set to zero. Moreover, in this latter case or if the cluster head flag changed, the receiver starts a decision process to resolve if it should or should not be a cluster head taking into account the new situation.

At any time, ITS stations can look at the timers in their databases to know how much time has passed since the last time they heard about the corresponding neighbors. If one of those timers exceeds a predefined amount of time, say $T_{\text {timer }}$, it is assumed that the corresponding ITS station is no longer a neighbor. In this case, the entry in the database is removed and the ITS station starts a new decision process. The value of parameter $T_{\text {timer }}$ should depend on the periodicity of the ITS message transmission and the ITS station speeds. In particular, it should be large enough to ensure the reception of at least one ITS message from every neighbor, and, at the same time, small enough to be able to adapt the cluster head selection to a rapidly changing VANET, i.e., one where the relative position of vehicles change rapidly.

The decision process is as follows: ITS stations become cluster heads if all neighbors have higher IDs or none of the neighbors with lower IDs is a cluster head.

\section{Remarks.}

1) With this technique, only one additional bit of information (the cluster head flag) is interchanged whenever an ITS message is transmitted. Note that this is true if some kind of ID is already included in the ITS messages, and, if not, the IDs should be also accounted as an extra payload.

2) When an ITS station starts a decision process, its database can be outdated. In particular, it may consider that a certain neighbor is a cluster head if this neighbor was not able to indicate a change in its state because no ITS message was received yet and the timer for this neighbor has not expired.

3) The databases can be reduced by including only the ITS stations with lower IDs.

\section{Probabilistic Cluster heAd SELECTION TECHNIQUE}

In this section, we propose a new type of cluster head selection techniques. The main advantage of these techniques is that they are able to combine different parameters that characterize the suitability of a cluster head (similarly to the weights of weight-based algorithms), maintaining the low signaling overhead of the LID technique (in contrast to weightbased algorithms which require the interchange of weights among other parameters). In particular, ITS stations decide to become a cluster head with certain probability computed from some parameters, and the decision is broadcasted using a onebit cluster head flag in the ITS messages. With this approach, ITS stations do not need to know the probability computed in neighboring stations.

Remarks.

1) A high (low) probability indicate a high (low) suitability of being a cluster head.

2) Due to the probabilistic nature of these algorithms, it is possible that ITS stations with high (low) probabilities decide to not become (become) cluster heads, although this possibility is unlikely depending on the actual probability values. This fact is, however, not critical for improving the timeliness of RHW message reception. In this case, it is more important to reduce signaling overhead and to allow certain overlapping of the cluster head coverage areas, which provides diversity.

3) In order to ensure that the signaling is composed of just one bit of information, i.e., the cluster head flag, the parameters used to compute the probability have to be already known by the ITS stations by either their own measurements or the content of ITS messages.

With a probabilistic cluster head selection technique, each ITS station should

1) choose a decision making interval $T$;

2) wait for $T$ seconds monitoring the channel;

3) after the $T$ seconds, compute a probability $P$ (see an example in Section IV-A) and become a cluster head with this probability; and

4) return to the first step.

The decision making interval, $T$, can be randomly selected from a predefined set of values, or it can be preconfigured in each ITS station. In any of the two cases, these intervals should be different in each ITS station to prevent their potential synchronization that may produce a ping pong effect, i.e., many ITS stations changing their role as cluster heads in every decision.

Key properties that the probability $P$ should exhibit are that (i) it should be 1 , i.e. $100 \%$, if no ITS message with a positive cluster head flag is received during the $T$ seconds, and (ii) it should decrease with the number and signal strength of these messages. By controlling how much the probability decreases, the overlap of cluster head coverage areas can be controlled. In particular, by using a method in which $P=1$ independently of the received flags, all ITS stations become cluster heads; and by using a method in which $P=0$ if one or more positive flags are received, cluster heads tend to be located outside the coverage areas of other cluster heads. This behavior is similar to the one of the LID-based technique of Section III, and, although it could be seen as a good property, the probabilistic behavior of fast fading and the rapidly changing vehicle locations produce coverage holes. That is to say, it is possible that an ITS station is out of the coverage of any cluster head. Since coverage holes are a serious problem, allowing some coverage overlap, e.g., with $P>0$, the formation of those coverage holes can be 
drastically reduced. In Section IV-A, a probability computation method with this behavior is proposed. This method can be mixed with the use of weights, as illustrated in Section IV-B.

\section{A. Distance-based probability of becoming a cluster head}

This section presents an example of how to compute the probability of becoming a cluster head depending on the distance to other cluster heads, which can be known from their ITS messages using either the signal strength or the Global Positioning System (GPS) information embedded in the message. Assuming that during the $T$ seconds of monitoring time an ITS station received positive cluster head flags from $N$ different vehicles, this ITS station should

1) estimate the distance to all senders, $d_{i}, i=1, \ldots, N$;

2) estimate the coverage range of its own VANET transmitter, $R$; and

3) calculate $P=\prod_{i=1}^{N}\left(\min \left(d_{i}, R\right) / R\right)$.

The coverage range, $R$, depends mainly on the environment, i.e., road or street shape, presence of objects that obstruct the signal, etc. Therefore, it should adapt to the particular environment in which the ITS stations are located. To this end, it could be estimated from the received ITS messages with GPS information, or from a testbed or simulation campaign and configured in the ITS stations as a fixed value for certain areas or locations.

\section{B. Using weights with the probabilistic cluster head selection}

The use of weights to select cluster heads is a common method found in the literature (e.g., [8], [9], [10], [13]), in which the cluster heads are those ITS stations with highest weights. However, the methods proposed in the literature involve the exchange of certain amount of control data in order to (i) compute the weights of each ITS station, and (ii) exchange the weights with other ITS stations to know which one should be the cluster head. In order to reduce the control signals, (i) the weights should be computed from the information sent within the ITS messages without injecting additional overhead, and (ii) the weights should modify the probability of becoming a cluster head in such a way that ITS stations with the highest weights are more likely to become cluster heads, so that no weight information exchange among stations is required. In particular, whenever an ITS station has to decide to become a cluster head, i.e., step 3 in Section IV, it should

1) compute a probability $P$;

2) compute a weight, $w \in[0,1]$; and

3) decide to become a cluster head with probability $P w$.

Note that the weights used in the literature are not typically in the range $[0,1]$. In this case, it would be necessary to delimit the weights by a maximum value and normalize them by this value. The weight $w$ modifies $P$ in a different way in each ITS station. In particular, if the ITS station is considered a good (bad) cluster head, the weight is close to $1(0)$. Some factors used in other works to affect the weight value are the number of neighbor vehicles [7], [10], distance to neighbors [10], speed [8], [9], [10], and Signal to Noise Ratio (SNR) of the cellular signal [13].

\section{Simulation ENVIRONMENT}

This section presents the simulation environments used to compare the distribution of RHW messages using a CUC, a CBC, and two HCVC; one with the LID-based cluster head selection technique of Section III and another one with the distance-based probabilistic cluster head selection technique of Section IV-A. The simulations focus on the DL part of the communication with the cellular network, i.e., the distribution of the RHW message from the base stations to the ITS stations. For the purpose of this paper, RHW messages are required to be distributed in the whole simulation area. The ITS stations communicate in the VANET using an IEEE 802.11p-based technology and with the cellular infrastructure using LTE.

\section{A. Description of the simulation scenarios}

We considered a motorway and an urban scenario. Two aspects of these scenarios affect the performance of the different alternatives: congestion of the cellular network and propagation conditions.

With respect to the cellular network, the two aspects are interrelated. In particular, the motorway scenario presents large cellular inter-site distances that produce high path losses and low Signal to Interference and Noise Ratio (SINR) values. Conversely, the urban scenario is more densely populated with cellular sites, and the SINR distribution is significantly better than in the motorway scenario, cf. Figure 5 in Section VI. This fact leads to a more efficient utilization of cellular resources in the urban scenario, i.e., less resources are required for transmitting the same amount of information. In addition to this, the cellular network has less resources per sector in the motorway than in the urban scenario, cf. Section V-E, due to the lower bandwidth availability in lower carrier frequencies. The use of these carriers in the motorway scenario is motivated by the typical LTE deployments in rural areas, which use lower frequencies because of coverage reasons. Due to the above, it is expected that the use of the HCVCs will be especially beneficial in the motorway scenario, since, in this case, the cellular network is expected to be easily saturated if a CUC is used.

With respect to the VANET, the propagation conditions influence the effects of hidden nodes, i.e., ITS stations whose transmissions cannot be detected by the transmitter and that collide and destroy the message at the receiver. These collisions affect the performance of the HCVC, the only configuration that uses the VANET to distribute RHW messages, cf. the fifth step in Figure 1. In particular, hidden nodes interfere with the distribution of RHW messages, CAMs and the embedded signaling bit, and hence, may affect the cluster head selection. The presence of hidden nodes is a particular aspect of the ITS-G5 technology, caused by the medium access control mechanism inherited from the IEEE 802.11 standard. In LTE, collisions may happen during the random access procedure when terminals in idle state have new data to transmit and demand resources. However, these collisions are more unlikely than in the case of ITS-G5 for two main reasons. First, the resource requests are significantly smaller than the ITS messages, cf. Section V-D. Second, the potential 


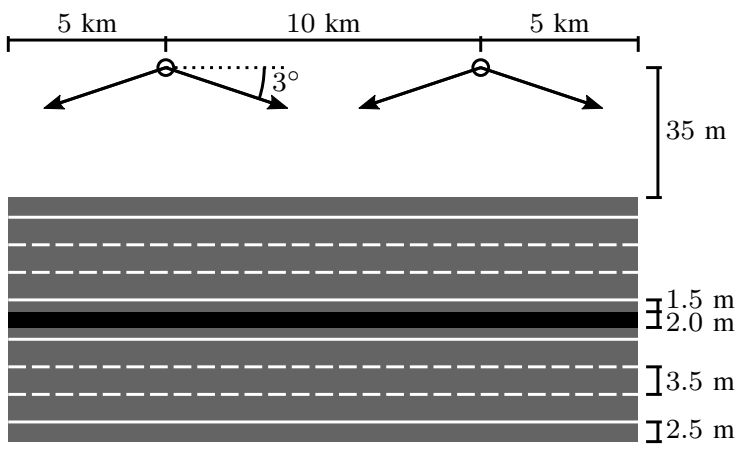

Fig. 2. Representation of the motorway scenario

colliding terminals are only new cluster heads, and not the entire set of ITS stations. For these reasons, collisions in LTE are assumed to be negligible in the results of Section VI. The propagation conditions of the motorway scenario lead to V2V links that are not significantly obstructed, which reduces, although not completely eliminates, the effects of the hidden nodes. Without significant obstruction, hidden nodes are generally located at long distances from the transmitter, and, in this case, their interference in the transmitter neighborhood is not destructive. Conversely, in the urban scenario, buildings significantly obstruct the V2V links. Due to this obstruction, hidden nodes can be located at short distances from the transmitter, which negatively impacts performance as shown in [15]. An illustrative example studied in [15] is the case in which, due to building obstruction, an ITS station may not know if the channel is being used by another ITS station in a perpendicular street, even if both stations are at a short distance. Therefore, the first station may initiate a message transmission that overlaps in time with the transmission of the second station. As a result, both transmissions interfere with each other in the street junction in such a way that a third vehicle could not receive any of the two messages.

Taking these conditions into account, it is expected that the HCVC will beat the CUC in the motorway, but not in the urban scenario, in terms of RHW message reception delay.

The motorway scenario, represented in Figure 2, is composed of a straight $20 \mathrm{~km}$ long motorway with three $3.5 \mathrm{~m}$ wide lanes per direction, a $2 \mathrm{~m}$ wide median, two $2.5 \mathrm{~m}$ wide external berms and two $1.5 \mathrm{~m}$ internal (beside the median) berms. Two cellular sites are located at $5 \mathrm{~km}$ and $15 \mathrm{~km}$ of one of the motorway ends, and separated $35 \mathrm{~m}$ from the motorway edge. The sites have two sectors, and an antenna height and downtilt of $20 \mathrm{~m}$ and $6^{\circ}$, respectively. With these parameters and assumptions, several simulations were carried out to obtain the best antenna azimuth for each sector. The best results were obtained using an antenna azimuth of $3^{\circ}$ with respect to the east direction, the motorway being eastwest oriented, for one sector, and the symmetric azimuth for the other sector.

The urban scenario, represented in Figure 3, is composed of a $7 \times 7$ Manhattan grid, i.e., vehicles can only be placed and move within a squared grid. The set of possible positions surrounds 49 regularly distributed square buildings of $22 \mathrm{~m}$ height and $125 \mathrm{~m}$ width. The streets are composed of four

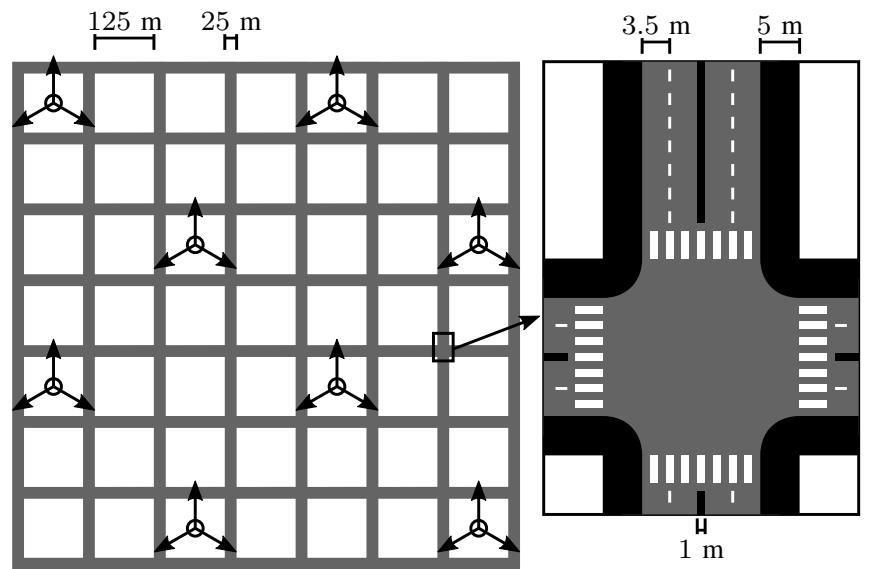

Fig. 3. Representation of the urban scenario

lanes, two in each direction, of $3.5 \mathrm{~m}$ width each, sidewalks of $5 \mathrm{~m}$ each, and a traffic island between both directions of $1 \mathrm{~m}$, resulting in a street width of $25 \mathrm{~m}$. Thus, the scenario is a square whose side length is $1075 \mathrm{~m}$ (7 buildings of $125 \mathrm{~m}$ and 8 streets of $25 \mathrm{~m}$ ). Eight cellular sites (each with three sectors) were located on the rooftop of the buildings shown in Figure 3.

\section{B. Propagation and channel models}

1) Cellular channel model in the motorway scenario: Path loss, shadowing and fast fading effects are simulated. The path loss model is based on the Rural Macro (RMa) model defined in the ITU-R specifications [16], in which different formulas are used depending on the Line of Sight (LoS) or Non-Line of Sight (NLoS) condition of cellular users. No user mobility is assumed in the RMa model, and a LoS or NLoS condition is selected for each user at the beginning of the simulation following certain LoS probability that depends on the distance to the base station. The selected condition is then fixed for the rest of the simulation. However, since, in this paper, ITS stations are moving, a modified version of this model was used. In particular, both the LoS, $L_{\mathrm{LoS}}$, and NLoS, $L_{\mathrm{NLoS}}$, path losses are weighted by the corresponding LoS probability, $P_{\mathrm{LoS}}$, i.e., the path loss at a distance $d, L(d)$, is

$$
L(d)=P_{\mathrm{LoS}}(d) L_{\mathrm{LoS}}(d)+\left(1-P_{\mathrm{LoS}}(d)\right) L_{\mathrm{NLoS}}(d),
$$

where

$$
\begin{gathered}
P_{\mathrm{LoS}}(d)= \begin{cases}1, & d \leq 10 \\
\exp \left(-\frac{d-10}{1000}\right), & d>10\end{cases} \\
L_{\mathrm{LoS}}(d)= \begin{cases}29.80+20.48 \log _{10}(d)+0.0014 d, & d \leq 502.65 \\
-22.23+40 \log _{10}(d), & d>502.65\end{cases} \\
L_{\mathrm{NLoS}}(d)=5.54+39.39 \log _{10}(d) .
\end{gathered}
$$

The equations for $P_{\mathrm{LoS}}(d), L_{\mathrm{LoS}}(d)$, and $L_{\mathrm{NLOS}}(d)$ were obtained following the indications in [16].

Correlated shadowing was simulated using a log-normal map with a standard deviation of $6 \mathrm{~dB}$ and a correlation distance of $100 \mathrm{~m}$ [17]. For each sector, a shadowing map was generated, ensuring that two close ITS stations experience 
TABLE II

TRAFFIC LIGHTS PATTERN IN THE URBAN SCENARIO

\begin{tabular}{|c|c|c|}
\hline Seconds & $\begin{array}{c}\text { Lights of } \\
\text { horizontal streets }\end{array}$ & $\begin{array}{c}\text { Lights of vertical } \\
\text { streets }\end{array}$ \\
\hline $0-30$ & green & red \\
\hline $30-35$ & yellow & red \\
\hline $35-45$ & red & red \\
\hline $45-75$ & red & green \\
\hline $75-80$ & red & yellow \\
\hline $80-90$ & red & red \\
\hline
\end{tabular}

similar shadowing values. The small-scale characterization is added on top of the large-scale effect using a tapped delay line channel model whose power delay profile is the Extended Vehicular A (EVA) profile, commonly used in 3GPP LTE studies [18].

2) Cellular channel model in the urban scenario: The used model is based on the METIS proposal for the Manhattan grid layout [19], with a minimum coupling loss of $70 \mathrm{~dB}$. The METIS model also comprises large and small-scale channel characterization. The former is implicitly modelled, together with path loss, thanks to its ray-based approach. The smallscale characterization is the same one used in the motorway scenario.

3) VANET channel model in the motorway scenario: In this case, the path loss model used for the motorway is based on the model proposed in [20]. According to this model, the path loss at a distance $d$ is calculated as

$$
L(d)=72.63+16 \log _{10} \frac{d}{10}+X,
$$

where $X$ is a zero-mean normally distributed variable with standard deviation of 4.4 that represents the shadowing in the large-scale model. This variable is spatially correlated according to an exponential decaying correlation, with a correlation distance of $20.5 \mathrm{~m}$ [20].

4) VANET channel model in the urban scenario: In this case, the selected channel loss model was the ITU-R Urban Micro (UMi) path loss model in [16], where both the transmitter and receiver heights are assumed to be $1.5 \mathrm{~m}$. This model differentiates three cases: propagation to the same street, to a perpendicular street and to a parallel street. According to [16], two different models are used for the first two cases, whereas the propagation losses are assumed to be infinite in the third case. In addition to these losses, we considered that vehicles located between the transmitter and receiver cause a $10 \mathrm{~dB}$ extra loss.

\section{Mobility models}

1) Motorway scenario: ITS stations were initially dropped uniformly distributed along the motorway lanes. ITS stations then moved, following the indications given in [21], with a constant speed of 100,120 or $180 \mathrm{~km} / \mathrm{h}$ depending on whether they are in the rightmost, center, or leftmost lane of their direction. Moreover, when ITS stations reach the end of the motorway, they re-appear at the start of the same lane and with the same speed.
2) Urban scenario: In this case, ITS stations moved at a maximum speed of $60 \mathrm{~km} / \mathrm{h}$, turned at street intersections with $50 \%$ of probability ( $25 \%$ left and $25 \%$ right), and stopped at red traffic lights or when the vehicle in front was within a distance of $4 \mathrm{~m}$. The traffic lights (in each street intersection, cf. Figure 3) switched simultaneously using the pattern shown in Table II which repeated every 90 seconds. The movement of vehicles was generated using the road traffic simulation package Simulation of Urban MObility (SUMO) [22].

\section{Traffic models}

1) Encapsulation and routing of RHW messages: The RHW messages were distributed using the Decentralized Environmental Notification (DEN) basic service [23] standardized for C-ITS. The notifications were encapsulated in DEN Messages (DENMs) of size 800 bytes. The DENM source is able to reach an ITS server using the cellular infrastructure. The server analyzes the DENM, decides a region in which it has to be distributed (the whole simulation area in this case), and sends the DENM to the cellular base stations in that region. We assumed that the DENM was received at the same time by all base stations.

2) Cooperative awareness messages: ITS stations periodically transmitted Cooperative Awareness Messages (CAMs) to neighbors in the VANET. In particular, every $100 \mathrm{~ms}$ the vehicles checked the following rules to know if a CAM had to be generated [24]:

- Generate CAM when absolute difference between current heading (towards North) and last CAM heading is greater than $4^{\circ}$.

- Generate CAM when distance between current position and last CAM position is greater than $4 \mathrm{~m}$.

- Generate CAM when absolute difference between current speed and last CAM speed is greater than $0.5 \mathrm{~m} / \mathrm{s}$.

If the time elapsed since the last CAM was greater or equal to 1 second, a new CAM was generated independently of the previous rules. Consequently, all the possible time intervals between CAM generations were $100 n$ ms, $n=1, \ldots, 10$.

The CAM size followed the indications in [25], i.e., the size is 209 bytes, plus 213 bytes in CAMs that carry a low frequency container (every $500 \mathrm{~ms}$ ), and plus 166 bytes in CAMs that carry a security certification (every second). The CAMs were used to transmit IDs, cluster head flags, and to compute distances to neighbors in the cluster head selection techniques of the HCVC.

Since the CAMs are not transmitted to and from the cellular network, they affect the performance of only the HCVC, and not that of the CUC and CBC. In particular, in the case of the HCVC, DENMs and CAMs are transmitted in the same channel band and, hence, can collide and compete for the channel. Moreover, they affect the cluster head selection since they carry relevant information for the selection techniques, as described in the previous paragraph.

3) Encapsulation of cluster head flags in cooperative awareness messages: In this section, we propose a method for encapsulating cluster head flags in CAMs. Although this 
method is not part of the standard, it is compatible with it. The CAMs are structured as follows [24].

- ItsPduHeader

- CoopAwareness

- GenerationDeltaTime

- Camparameters

* BasicContainer

* HighFrequencyContainer (HFC)

* LowFrequencyContainer (LFC), optional

* SpecialVehiclecontainer (SVC), optional

The standard defines different types of containers for the three containers in a CAM, i.e. HFC, LFC, and SVC. In particular, there are two types of HFC currently defined, only one type of LFC, and up to seven types of SVC. Each CAM may only include one of these container types.

The only LFC defined in the standard is the BasicVehiclecontainerLowFrequency (BVCLF), which is transmitted every $500 \mathrm{~ms}$. The proposed method defines a new LFC to carry the cluster head flag, which we called as ClusterheadVehiclecontainerLowFrequency (CVCLF). This type of container can be included in any CAM that does not contain a BVCLF.

In low mobility scenarios, the CAM generation rate can be reduced to one CAM per second. In those cases, every CAM should include one BVCLF, hence, an extra CAM is necessary to send the CVCLF. In spite of this extra message, this method is not expected to saturate the VANET, since, in this case, the CAM generation rate is very low.

\section{E. IEEE 802.11p and LTE configuration}

The configuration parameters of the different devices used in the simulation are presented in Tables III to V. In particular, the LTE base station, the IEEE 802.11p station, and the vehicle configuration parameters are given in Table III, Table IV and Table V, respectively. Note that, from Table III, the power per LTE resource block in the two scenarios is the same.

With respect to LTE, the LTE scheduler selects users and allocates resource blocks to them following a proportional fair criterion, as explained in [26], and giving maximum priority to the RHW messages. Thus, although other types of traffic are present in the simulations, consuming $80 \%$ of the LTE resources in average, this traffic only produces interference and does not compete against ITS traffic for resources. Most of the assumptions and parameters shown in Table III follow the ITU guidelines [16] and we refer to this document for further details.

In the case of CBC, LTE broadcast is used, i.e. eMBMS. In order to select a Modulation and Coding Scheme (MCS) for eMBMS, a coverage study was carried out by means of simulations to estimate the number of vehicles per sector that can be supported with each MCS. In particular, the coverage level for a given MCS was computed as the percentage of vehicles that are not in outage for this MCS. A vehicle was assumed to be in outage if it experiences more than 5\% BLER. The selected MCS was the highest one with a coverage value of at least $95 \%$. Following this process, QPSK with a coding
TABLE III

Configuration of LTE Base Stations

\begin{tabular}{|c||c|c|}
\hline & Motorway scenario & Urban scenario \\
\hline Bandwidth & $10 \mathrm{MHz}$ & $20 \mathrm{MHz}$ \\
\hline $\begin{array}{c}\text { Total transmit } \\
\text { power }\end{array}$ & $46 \mathrm{dBm}$ & $49 \mathrm{dBm}$ \\
\hline Carrier frequency & $800 \mathrm{MHz}$ & $2600 \mathrm{MHz}$ \\
\hline Sectorization & 2 sectors & $3 \mathrm{sectors}$ \\
\hline CBC MCS & $\begin{array}{c}\text { QPSK with } 0.44 \\
\text { coding rate }\end{array}$ & $\begin{array}{c}16 \mathrm{QAM} \text { with } 0.48 \\
\text { coding rate }\end{array}$ \\
\hline CBC MSP & $20 \mathrm{~m}$ ms & $25 \mathrm{~m}$ \\
\hline Antenna height & 2 transmit and 2 receive antennas \\
\hline $\begin{array}{c}\text { Number of } \\
\text { antennas per sector }\end{array}$ & 14 dBi & $17 \mathrm{dBi}$ \\
\hline Antenna gain & $70^{\circ}$ in horizontal plane and $10^{\circ}$ in \\
\hline $\begin{array}{c}\text { Antenna half } \\
\text { power beamwidth }\end{array}$ & \multicolumn{2}{|c|}{$16^{\circ}$} \\
\hline Antenna downtilt & 2 dB \\
\hline Cable loss & \multicolumn{2}{|c|}{} \\
\hline
\end{tabular}

TABLE IV

CONFIGURATION OF IEEE 802.11P STATIONS

\begin{tabular}{|c||c|}
\hline Bandwidth & $10 \mathrm{MHz}$ \\
\hline Total transmit power & $\begin{array}{l}28 \mathrm{dBm} \text { in urban scenario and } \\
23 \mathrm{dBm} \text { in motorway scenario }\end{array}$ \\
\hline Carrier frequency & $5900 \mathrm{MHz}$ \\
\hline MCS / data rate & QPSK with 0.5 coding rate / 6 Mbps \\
\hline EDCA access category & Voice \\
\hline
\end{tabular}

TABLE V

VEHICLE CONFIGURATION

\begin{tabular}{|c||c|}
\hline Antenna height & $1.5 \mathrm{~m}$ \\
\hline Number of antennas & 2 transmit and 2 receive antennas \\
\hline Antenna gain & $2 \mathrm{dBi}$ \\
\hline Antenna pattern & Omnidirectional \\
\hline Cable loss & $0.5 \mathrm{~dB}$ \\
\hline Implementation loss & $5 \mathrm{~dB}$ \\
\hline Noise figure & $7 \mathrm{~dB}$ \\
\hline Thermal noise level & $-174 \mathrm{dBm} / \mathrm{Hz}$ \\
\hline
\end{tabular}

rate of 0.44 was selected for the motorway scenario, and 16QAM with a coding rate of 0.48 for the urban scenario.

Another important parameter of eMBMS is the Multicast Channel (MCH) Scheduling Period (MSP). The lowest value for this parameter allowed in the standard is $80 \mathrm{~ms}$, which is the value used in this assessment in order to minimize the RHW message transmission latency. Note that, depending on the instant at which a RHW message is generated, it has to wait for the start of the following MSP, i.e., between 0 and $80 \mathrm{~ms}$. Assuming that the RHW message generation instant and the beginning of the MSPs are independent, the mean waiting time is $40 \mathrm{~ms}$.

With respect to the IEEE 802.11 p configuration, the total transmit power of the stations was optimized for the transmission of CAMs in both the urban and motorway scenarios. The optimization aims at maximizing the number of CAMs 
correctly received in the proximity of the stations.

The parameters summarized in Table $\mathrm{V}$ are assumed to be common to the LTE and IEEE 802.11p equipment on board the vehicles. Implementation losses are assumed to model the signal losses due to imperfections in the fabrication of the receiver components.

In all the configurations, we assume $9 \mathrm{~ms}$ minimum delay in the transmission of the RHW messages accounting for $5 \mathrm{~ms}$ in the transmission from the originating remote ITS server to the LTE base station, $2 \mathrm{~ms}$ of base station processing, and $2 \mathrm{~ms}$ of receiver processing.

\section{F. System level simulator}

The system performance assessment presented in Section VI is based on dynamic system level simulations performed on a $\mathrm{C}++$ proprietary simulator with an implementation of both LTE and IEEE 802.11p. The LTE part was used in the framework of the WINNER+ project [27], which was one of the International Mobile Telecommunications-Advanced (IMT-Advanced) evaluation groups of the ITU-R, and more recently in the METIS project in the evaluation of the $5 \mathrm{G}$ system [28]. The IEEE 802.11p part emulates both physical and link layers. With respect to the link to system abstraction, i.e., the error probability given an SINR value, the model in [29] was used for IEEE 802.11p, and the model in [26] for LTE.

\section{RESUlts}

In this section, we provide performance results of an IEEE 802.11p-based VANET and an LTE cellular infrastructure interoperating in a CUC, a CBC, and two HCVCs. The two HCVCs differ in the cluster head selection technique. In particular, one of the HCVC uses the LID-based technique of Section III with $T_{\text {timer }}=300 \mathrm{~ms}$ in the motorway scenario and $T_{\text {timer }}=1100 \mathrm{~ms}$ in the urban scenario. This configuration is referred to in the figures as HCVC-LID. The selected values for $T_{\text {timer }}$ follow the guidelines given in Section III. In particular, these values equal the longest CAM periodicity of the scenario plus an extra time of $100 \mathrm{~ms}$ to counteract small delays originated by the collision avoidance of the IEEE $802.11 \mathrm{p}$. The other HCVC, which is referred to as HCVC-PROB, uses the distance-based probabilistic technique of Sections IV and IV-A with a decision making interval $T$ equal to the period between consecutive CAMs, i.e., the decision of becoming a cluster head is made every time a CAM is sent. Due to the distinct CAM transmission periodicities in the different ITS stations, this configuration of the decision making interval is enough to avoid the ping pong effect. The coverage range $R$ of the IEEE 802.11p transmitters was estimated by means of simulations as the distance at which $95 \%$ of CAMs were successfully received in the lowest loaded motorway and urban ${ }^{1}$ scenarios. This resulted in $R=362 \mathrm{~m}$ in the motorway scenario and $R=150 \mathrm{~m}$ in the urban scenario. The performance of these configurations is measured with the

\footnotetext{
${ }^{1}$ In the case of the urban scenario, only vehicles in the same street (without building obstruction) were considered.
}

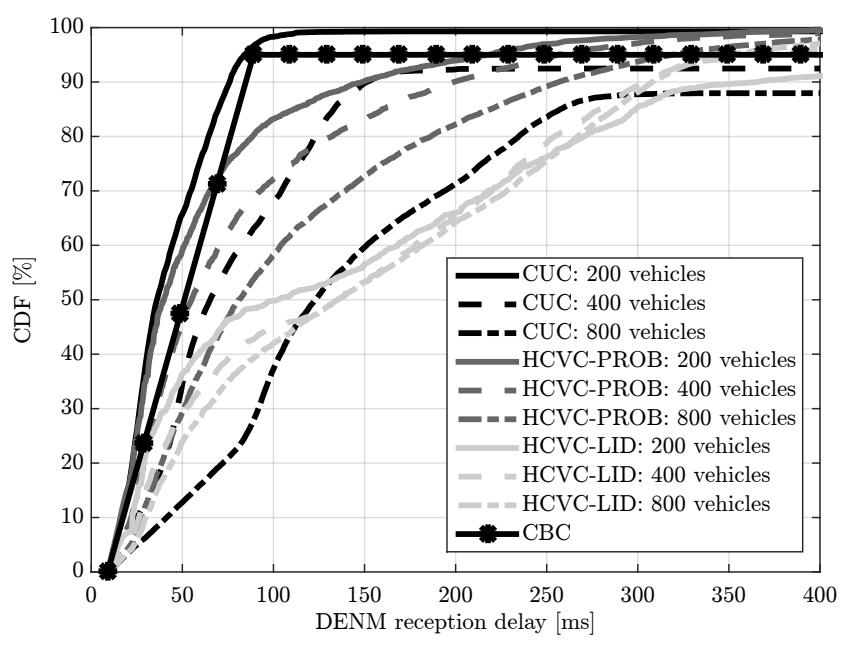

Fig. 4. CDF of the RHW message reception delay in the motorway scenario.

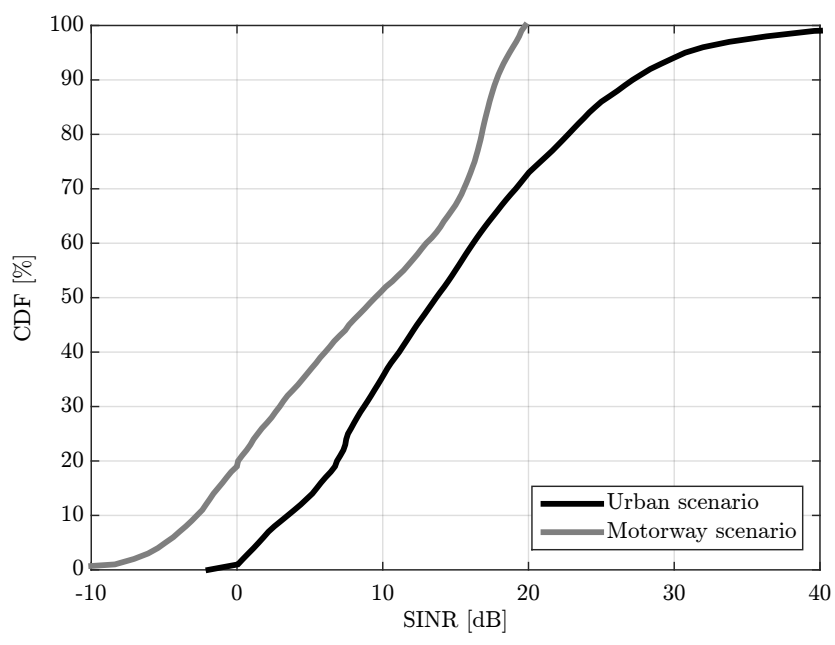

Fig. 5. CDF of the LTE SINR in the motorway and urban scenarios.

RHW message reception delay and the total amount of used LTE DL resources. These results are summarized in Table VI at the end of this section.

To ensure statistical significance of the results, multiple simulation runs (20 in the motorway scenario and 10 in the urban scenario) with different random seeds were performed.

\section{A. Motorway scenario}

Simulations in the motorway scenario were conducted for vehicle densities ranging from 20 to 800 vehicles on the motorway ( 5 to 200 vehicles per LTE sector), which is considered reasonable for this scenario.

1) Delay analysis: The CDF of the RHW message reception delay for three different quantities of vehicles $(200,400$ and 800) is depicted in Figure 4. According to this figure, CUC performance decreases drastically when the number of vehicles increases, and the probability-based HCVC, HCVCPROB, does not exhibit this drastic performance loss. In particular, although the CUC provides reduced delays compared to HCVC-PROB for 200 vehicles, the situation is reversed 


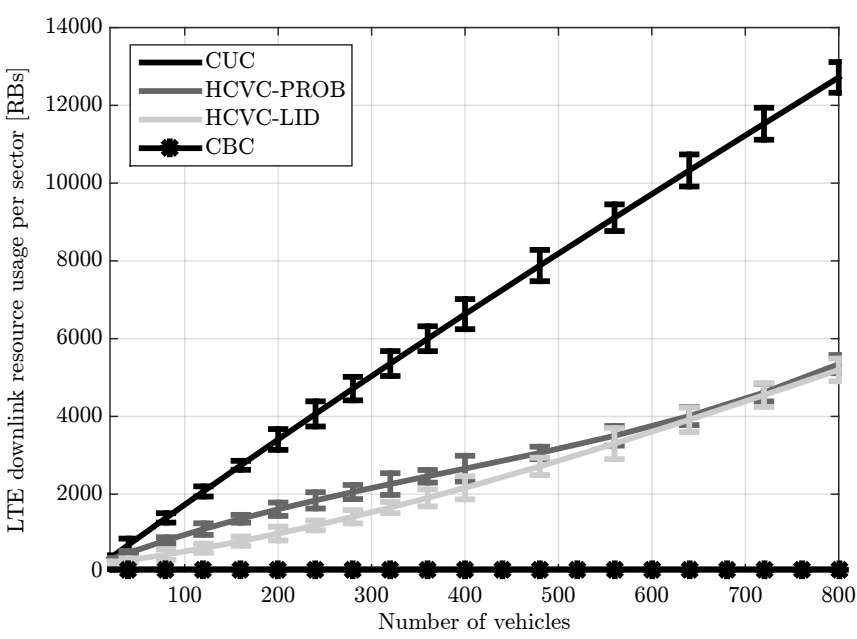

Fig. 6. Number of LTE resource blocks required per sector for the transmission of the RHW message in the motorway scenario.

when the number of vehicles is 800 . Moreover, it can be observed that, for the CUC with 400 and 800 vehicles, less than the $95 \%$ of the vehicles receive the RHW message within the first $400 \mathrm{~ms}$, whereas $320 \mathrm{~ms}$ is enough for HCVCPROB and all vehicle quantities. This behavior is due to the particular SINR distribution in this scenario, which exhibits a non-negligible probability of experiencing low SINR values, as shown in Figure 5. Specifically, the probability of experiencing a SINR lower than $-3 \mathrm{~dB}$, which is a typical acceptable SINR threshold [30, Table 6.16], is around a non-negligible $10 \%$. As a result, with the CUC, the low SINR values have to be compensated with more resources, which saturates the system and delays the RHW message distribution. With the probabilitybased HCVC, the vehicles located in low SINR zones can receive the RHW message from neighboring cluster heads. The CDFs of the LID-based HCVC, HCVC-LID, are very similar independently of the number of vehicles. However, these CDFs are close to the worst CUC CDF (for 800 vehicles), and hence, this configuration exhibits the worst performance. The CBC CDF does not depend on the number of vehicles (due to its broadcasting nature). In this case, the $\mathrm{CDF}$ reaches a maximum value of $95 \%$. This is a conservative assumption based on the QoS requirement used to select the MCS of eMBMS (95\% of locations with $95 \%$ of received packets). The CDF for $\mathrm{CBC}$ increases linearly from $9 \mathrm{~ms}$ (the minimum delay) up to $89 \mathrm{~ms}$ (the MSP value plus the minimum delay), since the RHW messages need to wait for the start of the following MSP as explained in Section V-E.

In summary, the $\mathrm{CBC}$ rises as the best option in terms of delay for 400 and 800 vehicles, followed by the probabilitybased HCVC. For low density scenarios, as seen with 200 vehicles, the CUC outperforms all the other options, as expected. Considering all vehicle quantities, the $\mathrm{CBC}$ is, in general, the preferred option, and in case the broadcasting features of the cellular network are not activated, the probability-based HCVC is the best configuration.

2) LTE resource usage analysis: The LTE resource usage is shown, together with confidence intervals, in Figure 6

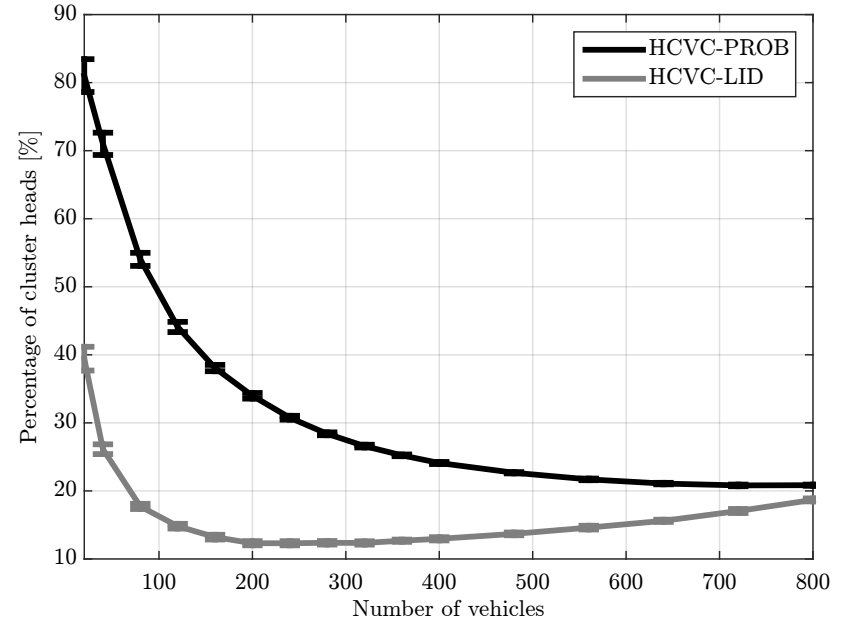

Fig. 7. Percentage of cluster heads in the motorway scenario.

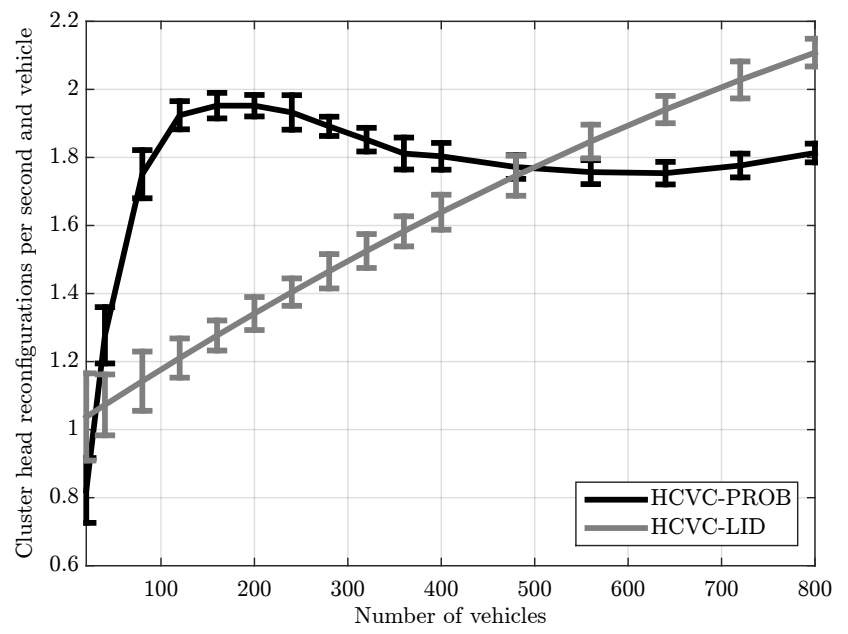

Fig. 8. Average number of cluster head reconfigurations (no cluster head to cluster head, and cluster head to no cluster head) per second and vehicle in the motorway scenario.

for all the considered configurations. The figure represents the number of resource blocks per sector needed to transmit the RHW message. In all configurations, the resource usage increases almost linearly with the number of vehicles. The slope of the HCVCs is significantly lower than that of the CUC, since the RHW messages are only sent to the cluster heads using LTE resources. The LTE resources savings make the HCVCs up to 2.5 times more efficient than the CUC. For the $\mathrm{CBC}$, the number of resources required to transmit a RHW message ( 862 bytes at the physical layer) with the selected MCS, cf. Section V-E, is about 69 resource blocks. Due to the broadcasting nature of the $\mathrm{CBC}$, this quantity of resources suffices to distribute the RHW message to $95 \%$ of vehicles in $89 \mathrm{~ms}$ (cf. Figure 4). This corresponds to the lowest resource usage of all compared options, and does not depend on the number of vehicles. The $\mathrm{CBC}$ is, therefore, again the preferred option. 
3) Cluster head selection analysis: In this section, the two cluster head selection techniques are compared in terms of the percentage of vehicles playing the role of cluster head and the cluster head reconfiguration rate, cf. Figures 7 and 8, respectively.

In both cases, i.e., the probability-based and the LIDbased HCVCs, the percentage of cluster heads in the scenario converges to around the $20 \%$ from higher initial values for low vehicle densities. The probability-based HCVC is always above the LID-based HCVC, due to the non-null probability of becoming a cluster head even with neighboring cluster heads.

The cluster head reconfiguration rate can be used as a measurement of the control traffic overhead between the vehicles and the ITS server due to the cluster head reselection signaling. In particular, a lower number of reconfigurations implies less overhead. This metric is influenced, among other factors, by the VANET interference level, which increases the CAM packet loss probability. Since the cluster head flag is embedded in the CAMs, the CAM losses imply that vehicles are unaware of some of their neighbors at the moment of the cluster head decision process, which may cause vehicles to make wrong decisions that have to be later reversed. In the case of this scenario, higher vehicle densities increase the quantity of transmitted CAMs and, hence, the VANET interference level. As shown in Figure 8, the probability-based HCVC is more stable than the LID-based HCVC with respect to this effect, since the cluster head reconfigurations converge to 1.8 reconfigurations per second and vehicle for the probabilitybased HCVC, whereas they increase linearly with the vehicle density for the LID-based HCVC. This result highlights the good performance of the probability-based HCVC with respect to the LID-based HCVC: it does not only distribute the RHW messages with lower latencies and consuming a similar quantity of LTE resources, but it also reduces the signaling overhead in LTE in highly dense scenarios.

Another factor that increases the VANET interference level in these simulations is the RHW message distribution through the VANET performed by the cluster heads. In particular, with more cluster heads, more interference is introduced to the VANET at the moment of the RHW message distribution. This fact makes that, due to the difference in the percentage of cluster heads shown in Figure 7, the reconfiguration rate increases faster in the region with low vehicle densities for the probability-based HCVC.

The number of reconfigurations under both techniques could be reduced by increasing the timer $T_{\text {timer }}$ (for the LID-based technique) and decision period $T$ (for the probability-based technique). However, in scenarios with high-speed vehicles like this one, this may increase the probability of creating cluster heads coverage holes.

\section{B. Urban scenario}

Simulations in the urban scenario were conducted for user densities ranging from 200 to 3500 vehicles ( 8 to 146 vehicles per LTE base station).

1) Delay analysis: The CDF of the RHW message reception delay for three different quantities of vehicles $(800,2400$

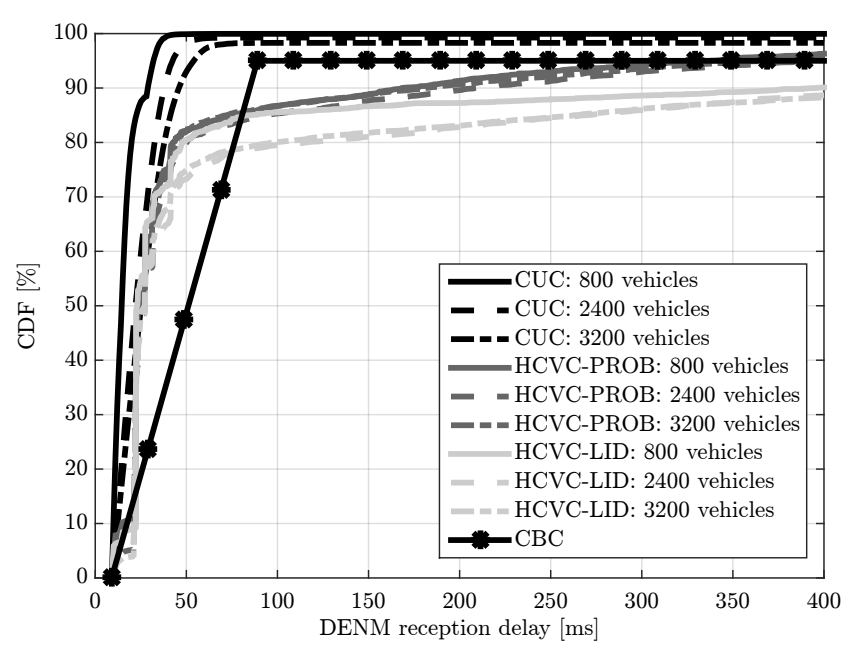

Fig. 9. CDF of the RHW message reception delay in the urban scenario.

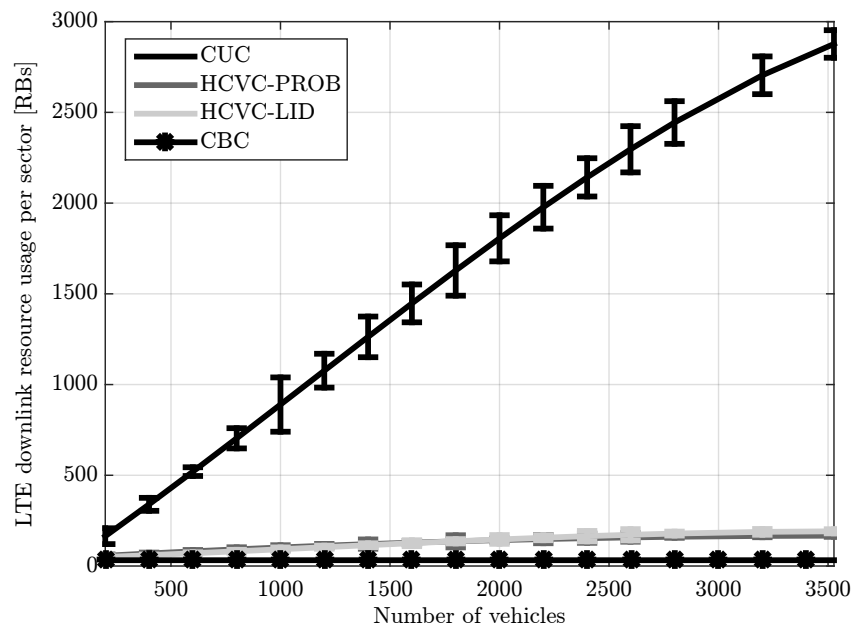

Fig. 10. Number of LTE resource blocks required per sector for the transmission of the RHW message in the urban scenario.

and 3200) is depicted in Figure 9. It can be seen that the CUC outperforms the other options in all considered cases, and the latency increase with the number of vehicles is very small. Therefore, in this case, the CUC is the preferred option. The better performance of LTE in the urban scenario compared to the performance in the motorway scenario is due to the availability of more resources and a better SINR distribution, as shown in Figure 5. The two studied HCVC techniques provide, in general, worse performance in terms of latency than the CUC, the LID-based approach being the worst one. Contrary to the motorway scenario, the obstruction of the V2V links caused by buildings in intersections increases the effects of hidden nodes, and, hence, impairs the two HCVC mechanisms. Nevertheless, it is important to note that at least $95 \%$ of the vehicles receive the RHW message within the first $400 \mathrm{~ms}$ with the probability-based HCVC.

2) LTE resource usage analysis: The LTE resource usage is shown in Figure 10 for all considered configurations. In this case, the HCVCs can be up to 14 times more efficient 


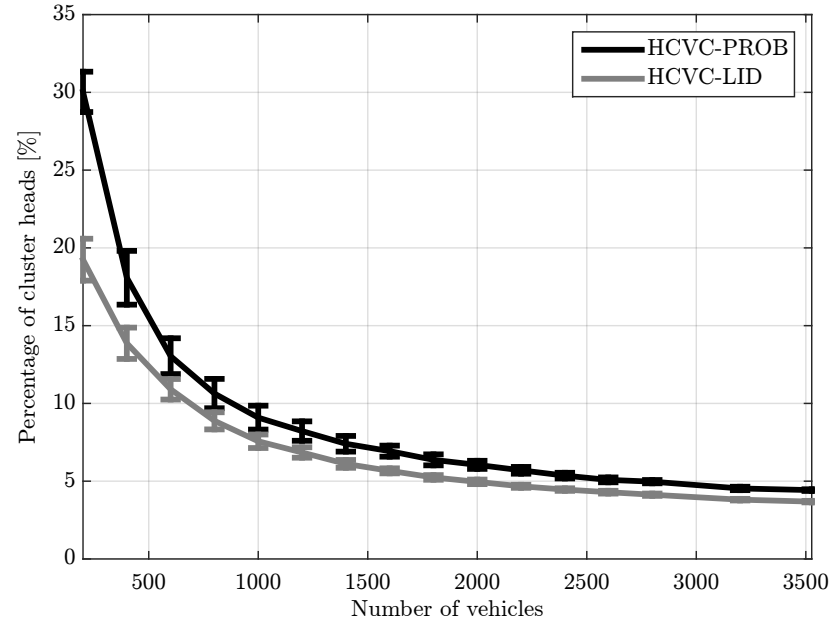

Fig. 11. Percentage of cluster heads in the urban scenario.

than the CUC, the resource usage of both HCVCs being quite similar. For the CBC, we considered that the RHW message transmission is conducted with 16QAM and coding rate 0.48 , as indicated in Section V-E. The number of resources required to transmit a RHW message ( 862 bytes at physical layer) with such MCS is about 32 resource blocks.

The good performance of the CUC in terms of delay, hence, comes at the price of a huge amount of consumed LTE resources, which may be prohibitive in some cases. Due to this, the CBC can be viewed, again, as the best option, or, if the broadcasting features of the cellular network are not activated, then the probability-based HCVC.

3) Cluster head selection analysis: Figure 11 shows the mean number of cluster heads in the HCVCs. The trend is similar for both approaches, but the probability-based HCVC is around 20 cluster heads above the LID-based HCVC. As compared with the motorway case, in the urban scenario, the percentage of cluster heads is much lower, stressing that the higher concentration of vehicles is well absorbed by the clustering approach.

Finally, Figure 12 shows the number of cluster head reconfigurations per second and vehicle. In the urban scenario, the vehicle speeds are lower than in the motorway scenario. This reduces the CAM generation rate, and hence, increases the cluster head decision intervals, which is reflected in lower cluster head reconfiguration rates, cf. Figures 8 and 12. In addition to this, in the urban scenario, the vehicle speeds depend on the vehicle density. In particular, due to the traffic lights and street intersections, the average vehicle speeds are lower with higher densities. This explains the lower slopes exhibited by the cluster head reconfiguration rate curves in the urban scenario as compared to the motorway scenario.

In this case, and due to a lower reconfiguration rate, the probability-based $\mathrm{HCVC}$ is the best cluster head selection technique.

\section{DISCUSSION}

The distribution of RHW messages to distant vehicles requires the VANET's interoperation with cellular networks due

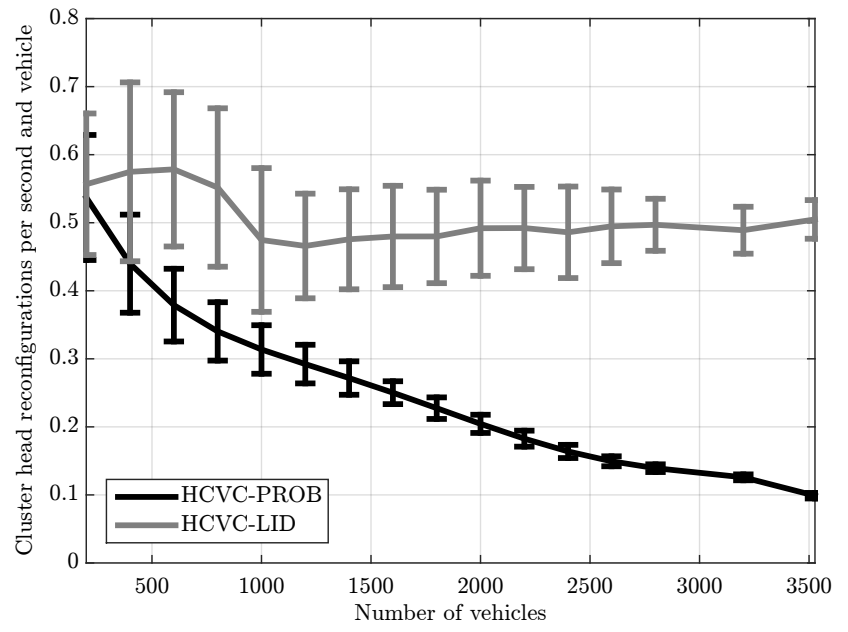

Fig. 12. Average number of cluster head reconfigurations (no cluster head to cluster head, and cluster head to no cluster head) per second and vehicle in the urban scenario.

TABLE VI

PERFORMANCE OF THE VANET AND CELLULAR INFRASTRUCTURE INTEROPERATION CONFIGURATIONS.

\begin{tabular}{|c||c|c|c|c|}
\hline & CUC & CBC & $\begin{array}{c}\text { HCVC- } \\
\text { PROB }\end{array}$ & $\begin{array}{c}\text { HCVC- } \\
\text { LID }\end{array}$ \\
\hline $\begin{array}{c}\text { Delay (motorway } \\
\text { scenario) }\end{array}$ & $\begin{array}{c}\text { medium } \\
\text { to high }\end{array}$ & low & medium & high \\
\hline $\begin{array}{c}\text { Delay (urban scenario) } \\
\text { low }\end{array}$ & medium & medium & $\begin{array}{c}\text { medium } \\
\text { to high }\end{array}$ \\
\hline consumption & high & $\begin{array}{c}\text { very } \\
\text { low }\end{array}$ & low & low \\
\hline
\end{tabular}

to the low efficiency of multi-hop transmissions. In this paper, the different interoperating configurations have been studied, namely Cellular Unicast Configuration (CUC), Cellular Broadcast Configuration (CBC), and Hybrid Cellular-VANET Configuration (HCVC). The results, which are summarized in Table VI, show that the sole use of a cellular LTE network in the distribution of RHW messages is an acceptable solution with either unicast or broadcast configuration as a better option depending on the scenario and vehicle density. In particular, the CBC of LTE is the most efficient configuration in terms of latency in a mid- to high-density scenario with reduced cellular capabilities (motorway scenario), and is the best one in all considered cases in terms of cellular resource consumption. In low-density scenarios and with high cellular capabilities (urban scenario), the CUC of LTE becomes the most efficient configuration in terms of RHW message latency, although it is the worst option in terms of cellular resource consumption. The hybrid schemes, based on the use of IEEE 802.11p with LTE support, present a reasonable trade-off of latency and cellular resource consumption in all considered cases. In particular, hybrid schemes consume significantly less cellular resources than LTE unicast, and can compete against LTE unicast and broadcast transmission modes in terms of latency, being the best option in high-density scenarios with reduced cellular capabilities.

The HCVC requires that some cluster heads are selected to 
act as gateways between the infrastructure and the VANET. However, cluster head selection techniques proposed in the literature are not designed for broadcasting RHW messages to distant vehicles with the support of infrastructure. Therefore, these techniques have certain drawbacks that have been analyzed in Section II. In order to alleviate them, a new alternative, in which cluster heads are selected with certain precomputed probability, has been proposed and compared with a new implementation of the LID-based. The main advantage of this alternative is that it combines different parameters that characterize the suitability of a cluster head, and, at the same time, requires only one signaling bit. As compared to the LID-based scheme, the new technique is more efficient in terms of latency and similar in terms of cellular resource consumption. Moreover, it exhibits a stable behavior regarding the reconfiguration of cluster heads, hence maintaining the signaling overhead under control and enabling the use of HCVCs.

Finally, it is important to highlight the relevance of hybrid schemes in market transition periods in which not all vehicles are equipped with both cellular and V2V connectivity as well as in the case of intermittent network coverage along the road. In these two scenarios, hybrid communication schemes might prove fundamental to ensure the availability of timely ITS data, and therefore, mark the future research lines on this topic.

\section{CONCLUSIONS}

In this paper, three VANET and cellular interoperation configurations to distribute RHW messages to distant vehicles have been analyzed. Despite distribution using cellular unicast or broadcast transmissions is a good option in terms of latency, the huge amount of resources required by unicast and the potential non-availability of broadcasting features in the cellular network make hybrid configurations a meaningful alternative. Indeed, they are strong competitors of unicast and broadcast in terms of both latency and resource usage.

The hybrid configurations require the selection of cluster heads to distribute the RHW messages. A new cluster head selection technique has been proposed in this paper to alleviate the drawbacks of previous techniques. The new scheme is able to reduce the latency of the RHW message distribution, and to select more stable cluster heads. These characteristics facilitate the use of hybrid configurations to distribute RHW messages.

\section{REFERENCES}

[1] ETSI TR 101607 V1.1.1, "Intelligent Transport Systems (ITS); Cooperative ITS (C-ITS); Release 1," May 2013.

[2] ETSI EN 302663 V1.2.1, "Intelligent Transport Systems (ITS); Access layer specification for Intelligent Transport Systems operating in the 5 GHz frequency band," July 2013.

[3] O. K. Tonguz, N. Wisitpongphan, and F. Bai,"DV-CAST: A Distributed Vehicular Broadcast Protocol for Vehicular Ad Hoc Networks," IEEE Wireless Communications, vol. 17, no. 2, pp. 47-57, 2010.

[4] K. Mershad, H. Artail, and M. Gerla, "We Can Deliver Messages to Far Vehicles," IEEE Transactions on Intelligent Transportation Systems, vol. 13, no. 3, pp. 1099-1115, 2012.

[5] J. Y. Yu and P. H. J. Chong, "A Survey of Clustering Schemes for Mobile Ad Hoc Networks," IEEE Communications Surveys \& Tutorials, vol. 7, no. 1, pp. $32-48,2005$.
[6] H. Wu, Z. Zhong, and L. Hanzo, "A Cluster-head Selection and Update Algorithm for Ad Hoc Networks," in Proc. Global Telecommunications Conference (GLOBECOM), 2010, Miami, Florida, USA.

[7] M. Gerla and J. T.-C. Tsai, "Multicluster, mobile, multimedia radio network," Wireless Networks, vol. 1, num. 3, pp 255-265, 1995.

[8] S. Basagni, "Distributed Clustering for Ad Hoc Networks," in Proc. 4th International Symposium on Parallel Architectures, Algorithms, and Networks (I-SPAN), 1999, Perth/Fremantle, Australia.

[9] S. Basagni, "Distributed and Mobility-adaptive Clustering for Multimedia Support in Multi-hop Wireless Networks," in Proc. IEEE VTS 50th Vehicular Technology Conference (VTC), 1999, Amsterdam, Netherlands.

[10] M. Chatterjee, S. K. Das, and D. Turgut, "WCA: A Weighted Clustering Algorithm for Mobile Ad Hoc Networks," Cluster Computing, vol. 5, num. 2, pp. 193-204, 2002.

[11] S. Baolin, C. Gui, Y. Song, and C. Hu, "Stable Clusterhead Selection Algorithm for Ad Hoc Networks," International Journal of Future Generation Communication and Networking, vol. 6, num. 3, pp. 95$105,2013$.

[12] M. A. Javed, D. T. Ngo, and J. Y. Khan, "A multi-hop broadcast protocol design for emergency warning notification in highway VANETs," EURASIP Journal on Wireless Communications and Networking, 2014:179.

[13] A. Benslimane, T. Taleb, and R. Sivaraj, "Dynamic Clustering-Based Adaptive Mobile Gateway Management in Integrated VANET - 3G Heterogeneous Wireless Networks," IEEE Journal on Selected Areas in Communications, vol. 29, no. 3, pp. 559-570, 2011.

[14] R. Sivaraj, A. K. Gopalakrishna, M. G. Chandra, and P. Balamuralidhar, "QoS-enabled group communication in integrated VANET-LTE heterogeneous wireless networks," in Proc. 2011 IEEE 7th International Conference on Wireless and Mobile Computing, Networking and Communications (WiMob), 2011, Wuhan, China.

[15] H. Tchouankem and T. Lorenzen, "Measurement-based Evaluation of Interference in Vehicular Ad-Hoc Networks at Urban Intersections," in Proc. from the 2015 IEEE International Conference on Communication Workshop (ICCW), 2015, London, United Kingdom.

[16] ITU-R M.2135-1, "Guidelines for evaluation of radio interface technologies for IMT-Advanced," December 2009.

[17] J. F. Monserrat, R. Fraile and L. Rubio, "Application of alternating projection method to ensure feasibility of shadowing cross-correlation models," Electronics Letters, vol. 43, no. 13, pp. 724-725, 2007.

[18] 3GPP TS 36.101 V8.0.0, "User Equipment (UE) radio transmission and reception (Release 8)," December 2007.

[19] METIS D6.1. Simulation Guidelines. Nov. 2013. https://www. metis2020.com/wp-content/uploads/deliverables/METIS_D6.1_v1.pdf.

[20] D. Vlastaras, T. Abbas, M. Nilsson, R. Whiton, M. Olbck and F. Tufvesson, "Impact of a Truck as an Obstacle on Vehicle-to-Vehicle Communications in Rural and Highway Scenarios," in Proc. IEEE 6th International Symposium on Wireless Vehicular Communications (WIVEC), 2014, Vancouver, Canada.

[21] CONVERGE Deliverable D4.3, "Architecture of the Car2X Systems Network," 2015. http://www.converge-online.de/?id=030000\&spid=en.

[22] Simulation of Urban Mobility (SUMO). http://sumo-sim.org/.

[23] ETSI TR 102 637-3 V1.1.1, "Intelligent Transport Systems (ITS); Vehicular Communications; Basic Set of Applications; Part 3: Specifications of Decentralized Environmental Notification Basic Service," September 2010.

[24] ETSI TS 102 637-2 V1.2.1, "Intelligent Transport Systems (ITS); Vehicular Communications; Basic Set of Applications; Part 2: Specification of Cooperative Awareness Basic Service," March 2011.

[25] CoCarX Deliverable D3, "ITS Services and Communication Architecture," 2012.

[26] D. Martín-Sacristán, J. F. Monserrat, V. Osa, and J. Cabrejas, "LTEAdvanced System Level Simulation Platform for IMT-Advanced Evaluation," Waves, vol. 3, pp. 15-24, 2011.

[27] WINNER+ IMT-Advanced Calibration: Guidelines, Software and Results. http://projects.celticinitiative.org/winner+/WINNER+ $\% 20$ Evaluation\%20Group.html.

[28] METIS Deliverable D6.5, "Final Simulation Results," 2015.

[29] M. Lacage and T. R. Henderson, "Yet Another Network Simulator," in Proc. from the 2006 Workshop on Ns-2: The IP Network Simulator, 2006, Pisa, Italy.

[30] A. ElNashar, M. A. El-saidny, and M. Sherif, "Design, Deployment and Performance of 4G-LTE Networks: A Practical Approach," Wiley, May 2014. 


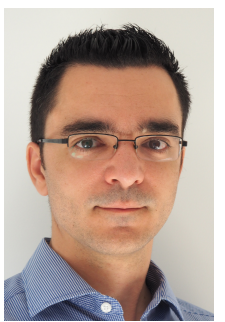

Daniel Calabuig (M'11) received the M.Sc. and $\mathrm{Ph} . \mathrm{D}$. degrees in telecommunications from the Universitat Politècnica de València (UPV), Valencia Spain, in 2005 and 2010 respectively. In 2005 he joined the Instituto de Telecomunicaciones y Aplicaciones Multimedia (iTEAM) from the UPV. During his $\mathrm{Ph} . \mathrm{D}$. he participated in some European projects and activities like NEWCOM, COST2100 and ICARUS, working on radio resource management in heterogeneous wireless systems and Hopfield neural networks optimization. In 2009 he visited the Centre for Wireless Network Design at the University of Bedfordshire, Luton, UK. In 2010 he obtained a Marie Curie Fellowship from the European Commission to research on cooperative multipoint transmissions. Thanks to this fellowship, Daniel Calabuig visited the Department of Systems and Computer Engineering at Carleton University, Ottawa, Canada, from 2010 to 2012. During 2012, he also visited the TOBB Ekonomi ve Teknoloji Üniversitesi, Ankara, Turkey. In 2012, he returned to the iTEAM and started working inside the European project Mobile and wireless communications Enablers for the Twenty-twenty Information Society (METIS), whose main objective is laying the foundation of $5 \mathrm{G}$, the next generation mobile and wireless communications system. He is currently involved in the METIS-II project.

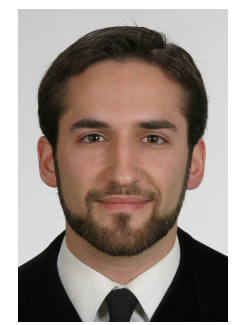

David Martín-Sacristán received his M.Sc. degree and $\mathrm{Ph} . \mathrm{D}$. degree in Telecommunications engineering from the Universitat Politècnica de València (UPV) in 2006 and 2016, respectively. Nowadays, he is a researcher in the iTEAM Research Institute of the UPV. He has been involved in several projects as a simulation expert, such as the European project WINNER+ that was an external evaluator of IMT-Advanced technologies for the ITU. He is currently participating in the European project METIS-II that leads the development of the 5G. His research interests are focused on the modeling and simulation of communication networks, radio resource management and vehicular communications.

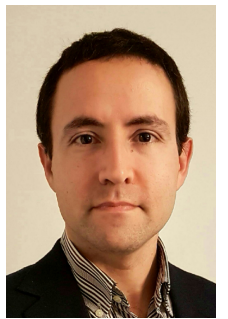

Jose F. Monserrat (M'03-SM'14) received his MSc. degree with High Honors and Ph.D. degree in Telecommunications engineering from the Universitat Politècnica de València (UPV) in 2003 and 2007, respectively. He was the recipient of the First Regional Prize of Engineering Studies in 2003 for his outstanding student record receiving also the Best Thesis Prize from the UPV in 2008. In 2009 he was awarded with the best young researcher prize of Valencia. He is currently an associate professor in the Communications Department of the UPV. His current research focuses on the design of future $5 \mathrm{G}$ wireless systems and their performance assessment. He has been involved in several European Projects, being especially significant his participation in NEWCOM, PROSIMOS, WINNER+ and METIS/METIS-II where he led the simulation activities. He also participated in 2010 in one external evaluation group within ITU-R on the performance assessment of the candidates for the future family of standards IMT-Advanced. He co-edited two special issues in IEEE Communications Magazine on IMT-Advanced and 5G technologies and is co-editor of the Wiley book "Mobile and wireless communications for IMT-Advanced and beyond" and the Cambridge book "5G Mobile and Wireless Communications Technology". Jose Monserrat is senior member of the IEEE, holds 6 patents and has published more than 40 journal papers. Currently the group headed by Prof. Jose F. Monserrat consists of 3 Postdoctoral fellows, 7 PhD students and 2 Master students.

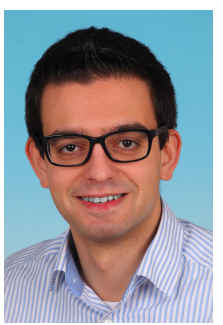

Mladen Botsov received his master's degree (M.Sc.) in electrical engineering and information technology from the Technische Universität München (TUM) in 2013. He is currently working towards his Ph.D. under the supervision of Prof. Dr.-Ing. habil. Slawomir Stanczak, Technische Universität Berlin. His research is centered on Device-to-Device (D2D) communications in $5 \mathrm{G}$ cellular networks. More specifically, his work examines radio resource management techniques for fast moving communication partners in a D2D underlay setup. Part of this work was conducted in the context of the European METIS project, to which he has been actively contributing between November 2013 and April 2015 .

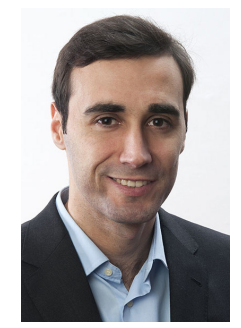

bile communication.
David Gozálvez received his M.Sc. and Ph.D. degrees in electrical engineering from the Universitat Politècnica de València (UPV) in 2007 and 2012, respectively. While working toward his Ph.D. degree, he was an active participant in the standardization process of digital video broadcasting - next generation handheld (DVB-NGH) as well as a contributor to the implementation guidelines of the second generation terrestrial digital video broadcasting (DVBT2). His research interests at present include future vehicular connectivity, specially focused on $5 \mathrm{G}$ mo- 